\title{
THE RELATIONSHIP BETWEEN TOOTH EXTRACTION AND CHANGES IN BLOOD MICROBIOME IN HORSES
}

\author{
A Thesis \\ presented to \\ the Faculty of the Graduate School \\ at the University of Missouri-Columbia \\ In Partial Fulfillment \\ of the Requirements for the Degree \\ Master of Science \\ by \\ KILE SHANNON TOWNSEND \\ Dr. Philip J. Johnson, Thesis Supervisor
}

MAY 2021 
The undersigned, appointed by the dean of the Graduate School, have examined the thesis entitled

\section{THE RELATIONSHIP OF TOOTH EXTRACTION AND CHANGES IN BLOOD MICROBIOME IN HORSES}

presented by Kile S. Townsend, a candidate for the degree of Master of Science, and hereby certify that, in their opinion, it is worthy of acceptance.

Dr. Philip J. Johnson

Dr. Aaron C. Ericsson

Dr. Elizabeth A. Giuliano

Dr. Carol R. Reinero 


\section{DEDICATION}

This thesis is dedicated to Chancey, the kind, fuzzy, energetic Morgan horse that started it all. Not only did you fascinate me and spark my love of horses, but you taught me how to ride, how to care for horses, and how to act as a mini veterinarian when I was a child. This thesis is also dedicated to all of the old, ailing horses of the world with dental disease. You are the horses that I grew up with and inspired me to become a veterinarian and, specifically, an equine internal medicine specialist.

Mom and Dad, you have supported my love of animals (specifically ponies), since day one and I often reflect upon how my upbringing was the best imaginable way to prepare me for my life as an educator and a veterinarian. Thank you for your love and support and for instilling in me the passion and drive to pursue and achieve goals at the highest level.

Erik and Nikolas, thank you for always having my back through thick and thin and for being excellent examples of what it is to be good human beings. From chatting about day-to-day life to proof-reading this thesis for me, I know that you are always there believing in me and in my potential. You inspire me to make a positive difference in the world each and every day.

Finally, I want to dedicate this thesis to my most loyal and loving sidekick, George the retired veterinary teaching beagle. From veterinary school to internship and then residency, you have been flopping by my side and making me smile. I am thankful for the long hours you have put in at the office, the clinic, in the lab, and at home on the couch to make this project and the beginning of my career a success. 


\section{ACKNOWLEDGEMENTS}

I would like to acknowledge all of my supporters, encouragers, and believers. I could not have completed this project without the unwavering support and enthusiasm of Dr. Johnson. You have inspired me to create a project that was not only doable, but meaningful, educational, and impactful. You have been so kind to encourage me to think critically, with common sense, and outside of the box throughout my residency. Your thousands of hours spent training me have been so appreciated and I could not have asked for a better advisor and mentor in veterinary medicine. I really could not have dreamed this project up without you. Dr. Johnson, you are in large part the reason I am going be a successful internist and academician!

Thank you, Dr. Ericsson, for your interest and energy that you have injected into this project. I am so thankful to have been able to use your lab and resources to get this project done. Your encouragement for publication and help with structuring and conducting this Master's project has been absolutely instrumental and indispensable.

Dr. Giuliano, I know this project was initially supposed to be about eyes and somehow evolved to teeth. Thank you for sticking with me and for your generous words of encouragement and guidance in this process. I have always appreciated your support of me during this residency program and have tremendously enjoyed working together.

Dr. Reinero, thank you for helping to shape this project into what it has become and guiding me to write grants, abstracts, and to make an impact in the scientific community. 
Dr. LaCarrubba, you have been integral to this project and I will always smile when reflecting on how many teeth we have successfully extracted together. You are the leverage to my gingival elevation and this project definitely would not have happened without your blood, sweat, and tears. Thank you, I love working (and riding) together! Thank you all for guiding me, encouraging me, and keeping me on the right path to defending this thesis. I appreciate all of the time, effort, and belief that you have all put into me and the project, it has not gone unnoticed - I am so thankful for it all. 


\section{TABLE OF CONTENTS}

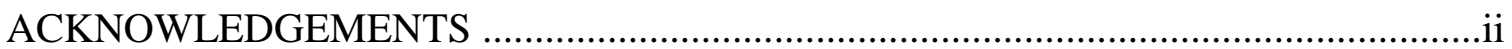

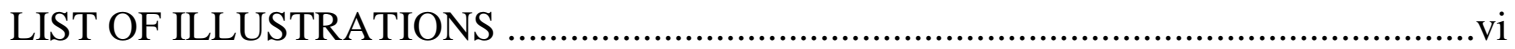

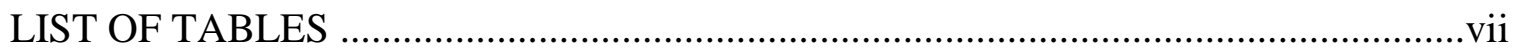

LIST OF ABBREVIATIONS .............................................................................viii

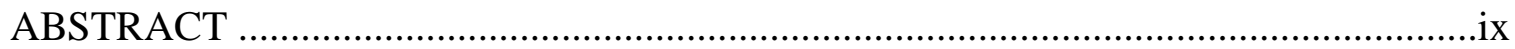

CHAPTER 1. INTRODUCTION ......................................................................

Oral Cavity Microbiome, Dental Disease, and Systemic Implications ..................

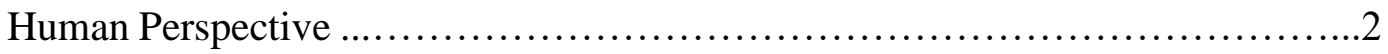

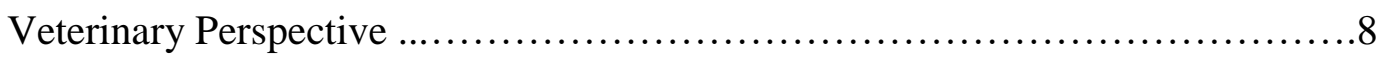

Next Generation Sequencing to Characterize Microbiomes and Uncultivable Bacteria ....10

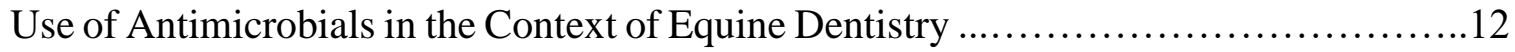

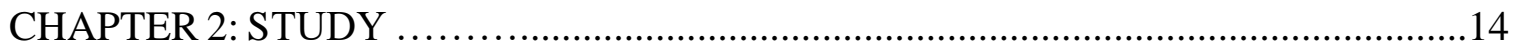

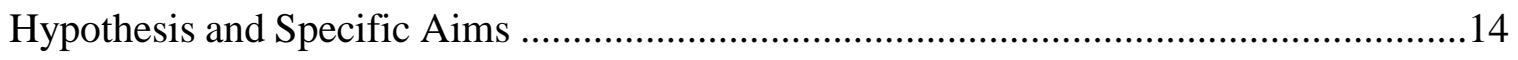

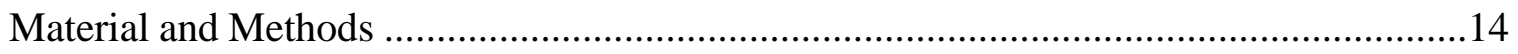

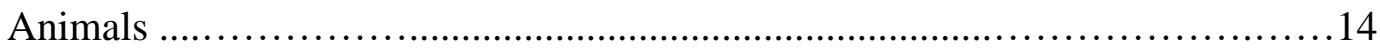

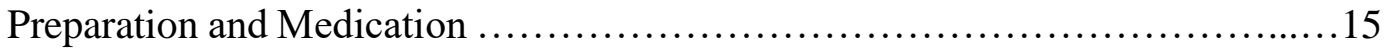

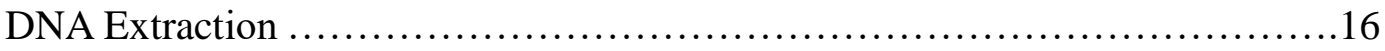

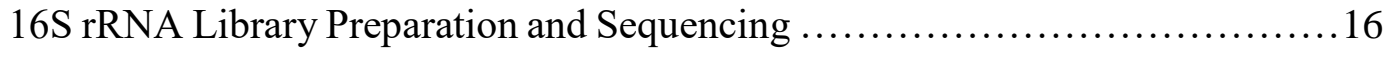

Bioinformatics Analysis ........................................... 17

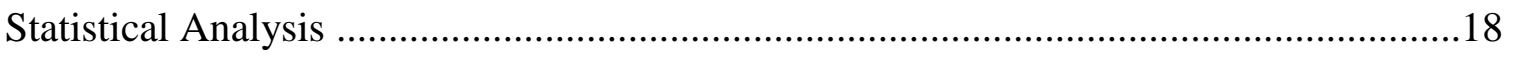

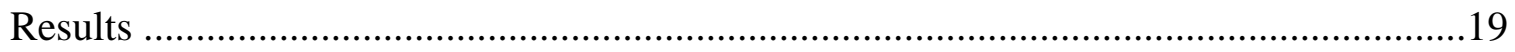

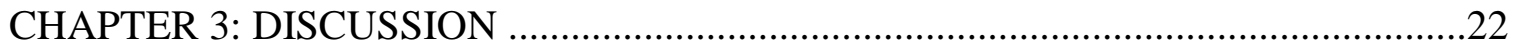

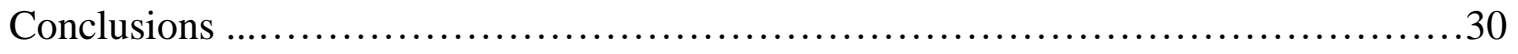


ILLUSTRATIONS

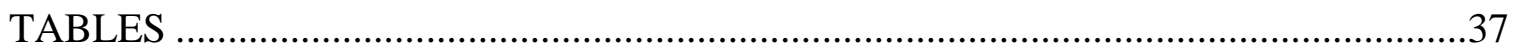

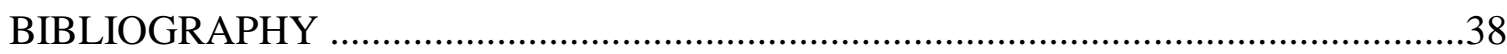




\section{LIST OF ILLUSTRATIONS}

Figure 1: Dot plots showing the total number of $16 \mathrm{~S}$ rRNA amplicon sequences resulting from amplification and sequencing on a shared flow cell, of negative (-) and positive (+) controls, peripheral blood collected aseptically pre- and post-

exodontia procedure, and dental/gingival swabs.

Figure 2: Bar chart showing sample coverage in those samples yielding $>1054$ sequences, legend at top (A), and a dendrogram generated from those data, rarefied to a uniform coverage of 1,054 sequences/sample (B)................36

Figure 3: Principal coordinate analysis based on Jaccard similarities and generated using a rarefied dataset $(1,054$ sequences/sample $)$

Figure 4: Heatmap generated via hierarchical clustering of samples based on the relative abundance of the 50 ASVs yielding the lowest $p$ values following ANOVA of all ASVs comparing pre- and post- exodontia blood and swabs .38

Figure 5. Pie chart showing the mean relative abundance of ASVs detected in the gingival swabs, with dominant genera labeled. The grey portion represents a total of 8,544 rare ASVs, comprising roughly $10 \%$ of any given sample. 


\section{LIST OF TABLES}

Table 1: Table depicting individual horse information including teeth extracted,

diagnosis, surgical procedure, and health designation (healthy, affected with PPID, equine asthma, sinusitis). Teeth were numbered using the Modified Triadan System. ${ }^{\mathrm{a}}$ refers to the first visit, ${ }^{\mathrm{b}}$ refers to second visit.....................40 


\section{LIST OF ABBREVIATIONS}

\begin{tabular}{|c|c|}
\hline ANOVA & Analysis of Variance \\
\hline ASV & Amplicon Sequence Variants \\
\hline BWT & Body Weight \\
\hline $\mathrm{CFU}$ & Colony Forming Units \\
\hline CRI & Constant Rate Infusion \\
\hline CVID & Common Variable Immunodeficiency \\
\hline DNA & Deoxyribonucleic Acid \\
\hline dNTP & Deoxynucleoside Triphosphate \\
\hline dsDNA & Double-Stranded Deoxyribonucleic Acid \\
\hline EB & Elution Buffer \\
\hline EDTA & Ethylenediaminetetraacetic Acid \\
\hline EOTRH & Equine Odontoclastic Tooth Reabsorption and Hypercementosis \\
\hline GC & Guanine-Cytosine \\
\hline IV & Intravenous \\
\hline NGS & Next Generation Sequencing \\
\hline PCoA & Principal Coordinate Analysis \\
\hline PCR & Polymerase Chain Reaction \\
\hline PERMANOVA & Permutational Multivariate Analysis of Variance \\
\hline PPID & Pituitary Pars Intermedia Dysfunction \\
\hline RNA & Ribonucleic Acid \\
\hline rRNA & Ribosomal Ribonucleic Acid \\
\hline SD & Standard Deviation \\
\hline
\end{tabular}




\title{
THE RELATIONSHIP OF TOOTH EXTRACTION AND CHANGES IN BLOOD
} MICROBIOME IN HORSES

\author{
Kile Shannon Townsend \\ Dr. Philip J. Johnson, Thesis Supervisor
}

\begin{abstract}
Bacteremia resulting from dental surgery is increasingly recognized as a health risk, especially in older and immunocompromised patients (in human medicine and also now in equine veterinary medicine). Dentistry-associated bacteremia can lead to remote infections, as exemplified by valvular endocarditis. Emerging evidence points to a novel role played by oral cavity commensals in the pathogenesis of diabetes, respiratory disease, cardiovascular disease, and adverse pregnancy outcomes. Whether dental extraction, a commonly undertaken procedure in old horses, causes bacteremia has not been reported extensively. In a prospective clinical study using next generation sequencing (based on bacterial 16S rRNA), the circulating blood microbiome was characterized before and at 1 hour following extraction of incisor, canine or cheek teeth from 29 adult horses with dental disease. 16S rRNA gene sequencing results from the blood microbiome were compared with those from gingival swab samples obtained prior to extraction at the location of the diseased tooth.
\end{abstract}

Univariate data were first tested for normality using the Shapiro-Wilk method. Non-normally distributed data were then tested using a Kruskal-Wallis analysis of variance (ANOVA) on ranks, followed by post hoc pairwise comparisons using Dunn's method, with significance defined by $\mathrm{p}<0.05$. Multivariate data were compared using permutational multivariate ANOVA (PERMANOVA) based on Jaccard similarities, using Past3 software. Bacteremia associated with translocated gingival commensals was 
demonstrated in horses undergoing exodontia and was, in some cases, still evident one hour post-operatively. 


\section{CHAPTER 1: INTRODUCTION}

\section{Oral Cavity Microbiome, Dental Disease, and Systemic Implications}

In human medicine, it has long been accepted that there exists a relationship between dental disease and systemic health. There have been numerous reports over the last 50 years of periodontal disease and its pathophysiological association with conditions such as diabetes mellitus, atherosclerotic cardiovascular disease, metabolic syndrome, chronic diseases (i.e. rheumatoid arthritis, cancer, respiratory diseases, Alzheimer's disease), and adverse pregnancy outcomes [1]. Recently, veterinarians have also begun to recognize and characterize pathophysiological associations between systemic health and periodontal disease in canine and feline species [2]. These relationships have led to broader recognition of the importance of routine oral examination and preventive hygiene in both human medicine and veterinary medicine.

Domestic animal species and humans commonly suffer from both gingivitis and periodontal disease. The prevalence of periodontal disease in dogs has been estimated at approximately $80 \%[3,4]$. In human medicine, mild periodontitis affects about $50 \%$ of adults and increases to over $60 \%$ in people over 65 years of age [1]. Periodontitis in its severe form is the sixth most common human disease (estimated to affect $11.2 \%$ of the global adult population) [5]. As dental disease has come into the spotlight in human and small animal medicine, substantial advances in veterinary dentistry and preventive health care have also been made in the specific field of equine dentistry. Although equine dental abnormalities and ailments have been recognized since antiquity, it was not until approximately the last 15 years that there have been major developments in routine examinations and in both simple and advanced dentistry procedures in this species [6]. 


\section{Human Perspective}

There exists a complex relationship between inhabitant microbiota of the oral cavity, dental/gingival disease, and systemic health. The healthy oral microbiome is an important part of the overall human microbiome and is comprised of several hundred to several thousand diverse species. As a normal component of the oral cavity, the oral microbiome functions to protect against colonization by extrinsic pathogenic bacteria that could cause local disease and adversely compromise systemic health [7]. It is now widely recognized that abnormal bacterial influence is central to the pathogenesis of the most commonly encountered oral diseases in people, including gingivitis, periodontitis, and dental caries [8].

The earliest recognition of an oral microbiome is attributed to Antony van Leeuwenhoek. Using a microscope of his own design, he reported that a diverse array of microorganisms is present in plaque that develops on dental surfaces and, remarkably, he deduced that individual differences in these microbial populations were related to the health of the oral cavity [8]. It is now recognized that oral microorganisms are organized into biofilms and that greater than 500 bacterial species inhabit the oral cavity, constituting the oral microbiome [9-11]. Biofilm is an aggregate of microorganisms in which cells adhere to each other and/or to a surface.

In general, dental disease (gingivitis, periodontitis, and caries) results initially from the development and advancement of dental plaque (also known as microbial plaque, oral biofilm, dental biofilm, dental plaque biofilm, or bacterial plaque biofilm) [12]. Disease initiation and propagation results from a dysbiosis of the commensal oral microbiota (dental plaque), which then interacts with host immune defenses and leads to 
inflammation and disease. For example, caries (dental decay) is a disease of the hard tissues of the teeth resulting from an imbalance in the interactions between cariogenic bacteria in dental plaque and fermentable carbohydrates (mainly sugars) that are retained in the mouth from the diet [13]. Disease prevention is facilitated by daily self-performed oral hygiene (tooth brushing and flossing) and regular professional removal of accumulated microbial biofilm (scaling performed by a dentist) [12].

Dental plaque is a biofilm or aggregation of bacteria that normally accumulates on hard surfaces within the oral cavity. It develops on interdental, lingual/palatal, and buccal/labial surfaces of teeth, including chewing surfaces, along the gum line, and below gum line margins [14]. In the healthy state, plaque is present as a thin layer that is continuously being restricted by the natural cleaning activities that result from chewing, saliva production, and physical actions of the tongue. In the absence of sufficient and effective cleaning or saliva production, plaque accumulates and becomes mineralized (calculus).

Formation of a bacterial plaque biofilm on newly erupted or cleaned dental surfaces is preceded by formation of pellicle, a layer of saliva that is composed mainly of glycoproteins that provide an adhesive role. Bacteria then attach to the pellicle layer, forming microcolonies, and subsequently maturing into a dental plaque biofilm. In excess of five hundred distinct bacterial species have been identified in dental plaque. The composition of dental surface microbiota is variable; different bacterial species are found during early surface colonization (early colonizers) compared with those identified in later stages of disease (late colonizers) [15]. Early colonizing bacteria include predominantly Streptococcal species (especially Streptococcus mutans) and other 
anaerobes (Fusobacteria and Actinobacteria); late colonizing species include Actinobacillus spp., Prevotella intermedia, Eubacterium spp., Treponema spp., Porphyromonas gingivalis, and Fusobacterium nucleatum [16]. Specific microbial biofilm composition also varies by location within the oral cavity.

Biofilm bacteria are normally found in the healthy oral cavity and are usually harmless. Supragingival biofilm, plaque that develops above the gums, is the first kind of plaque to form following dental brushing. Supragingival biofilm is comprised of mostly aerobic bacteria, needing oxygen to survive [14]. Anaerobic bacteria grow in (deeper) supragingival biofilms that remain on dental surfaces for longer periods of time. Biofilm bacteria in closest proximity to dental surfaces generally obtain energy through fermentation of dietary sucrose, leading to the production of acids [15].

Subgingival biofilm is plaque that is located under the gums. Subgingival biofilm develops after establishment of supragingival biofilm through local extension. Subgingival biofilm plaque is predominantly comprised of anaerobic bacteria; bacteria that only survive in the absence of oxygen. The subgingival location serves to protect these anaerobes from oxygen. The major local ecological factors within the oral cavity that influence and contribute to plaque formation include $\mathrm{pH}$, saliva, temperature, and redox reaction status $[17,18]$. In health, the natural oral cavity environment supported by saliva is optimal for bacterial growth in dental plaque [19].

Unfettered progression and build-up of a thick layer of dental plaque and calculus represent the principal causes for the common human dental diseases including gingivitis, periodontitis, and dental caries. Plaque removal and control is routinely achieved by regular tooth brushing and the employment of interdental cleaning devices such as dental 
floss and interdental brushes. Accumulated calculus cannot be removed by tooth brushing and can only be removed through professional cleaning (scaling) [20].

Gingivitis is the result of a host inflammatory reaction to local, excessive biofilm bacteria. In its early stages, gingivitis itself can be neutralized by simple plaque removal. However, if plaque accretion is unrecognized or untreated, gingivitis progresses to degradation of local supporting tissues, causing periodontitis. Plaque bacteria release enzymes that cause local osteolysis and promote establishment and progression of periodontitis [20].

Disruption of the gingival-blood barrier as a result of plaque biofilm bacteriainduced gingivitis/periodontitis potentially facilitates the translocation of bacteria and bacterial products into the circulation, which could lead to various systemic diseases [21]. Various diverse human diseases that have been attributed to this phenomenon have included adverse pregnancy outcomes, diabetes mellitus, respiratory disease, cardiovascular disease, and atheroma [22-24]. Antibodies to the oral pathogens Campylobacter rectus, Veillonella parvula, Prevotella melaninogenica have been specifically associated with hypertension [25].

Fusobacterium nucleatum is a fastidious Gram-negative anaerobe and an emerging pathogen for which laboratory cultivation is challenging. However, using culture-independent methods, such as Next Generation Sequencing (NGS) technology, an emerging role for this common plaque biofilm inhabitant in numerous human systemic diseases (adverse pregnancy outcomes [chorioamnionitis, preterm birth, stillbirth, neonatal sepsis, and preeclampsia], gastrointestinal disorders [colorectal cancer, inflammatory bowel disease, and appendicitis], cardiovascular disease, rheumatoid 
arthritis, respiratory tract infections, Lemierre's syndrome, and Alzheimer's disease) is becoming increasingly apparent [24]. Whereas $F$. nucleatum is regarded as ubiquitous in the oral cavity, it is absent or infrequently detected elsewhere in the body under normal conditions $[26,27]$. However, under disease conditions, $F$. nucleatum is one of the most prevalent species found at extra-oral sites [28]. The role of $F$. nucleatum in some of the conditions noted above is presently at the stage of association and proven cause-andeffect is yet lacking.

Dental caries is an infectious disease caused principally by Streptococcus mutans. Organic acids released from dental plaque bacteria cause demineralization of the adjacent dental surface, and consequently promote dental caries. The development of dental caries is predominantly dependent on the availability of fermentable sugars, other local environmental conditions, bacteria, and host factors. Although current understanding regarding microorganisms involved in the initiation and progression of caries is incomplete, the most relevant acidogenic-aciduric bacterial species recognized presently are Streptococcus mutans, bifidobacteria, and lactobacilli [29]. Mutans streptococci are initiators of disease progress and bifidobacteria and lactobacilli facilitate disease progression. Enhancing factors for pathogenic microbial activity include specific environmental conditions, such as the presence of fermentable dietary sugars and the absence of oxygen [29]. The present-day western diet is particularly cariogenic, not just because of the high amount of fermentable carbohydrates but also because food intake occurs with high frequency throughout the day [16].

Oral sepsis and oral or maxillofacial surgery (including exodontia) have been recognized as risk factors for bacteremia and endocarditis in human medicine since the 
1930s [30, 31]. Because of the close association of teeth to the gingival capillaries, it is logical to deduce that some bacteria located in the periodontal space could move directly into the bloodstream when the area is disrupted (by disease or surgery).

In human medicine, bacteremia has been confirmed as a potential sequel to dental treatment and is even associated with routine daily activities such as chewing of hard foodstuffs or tooth brushing [32]. The risk of developing bacteremia is reported to be as high as $100 \%$ following exodontia procedures in human patients [33]. Usually, bacteremia, particularly if it occurs during ordinary activities, does not result in complicating infections because those bacteria typically are present only in small numbers and are rapidly removed from the bloodstream by an effective immune system. It has been reported that bacteremia experienced under those circumstances results from only a low number of bacteria and is only present for up to 30 minutes [33]. However, if bacteria are present for long enough and in large enough numbers, particularly in people who have a weakened immune system, bacteremia can lead to other infections (various locations) and sometimes trigger a serious body-wide response (systemic inflammation and sepsis) [32]. Risk of transient bacteremia in people is related to both oral cavity bacterial load and to the severity of inflammation (gingivitis/periodontitis) in the oral cavity.

Transient bacteremia is especially important for patients with generalized poor health, underlying immunodeficiency, or other comorbidities because dental procedures may cause severe complications in this population [32]. Antimicrobial treatment is specifically recommended for people undergoing dental procedures that may cause bleeding, and therefore allowing overt ingress of bacteria into the bloodstream and 
circulation [32]. Other medical conditions warranting antimicrobial prophylaxis in humans could potentially be relatable to equine or veterinary patients as a whole. A few of those diseases and populations of patients include those affected with: endocarditis, obstructive cardiomyopathy, glomerulonephritis, labile diabetes mellitus, cancer, previous adverse pregnancy outcomes, and patients undergoing immunosuppressive treatment $[1,26,32]$.

\section{Veterinary Perspective}

Due to developing of interest in equine dentistry and preventive medicine for horses, it has been recognized that many of the same dental conditions that afflict small animal species and humans are present in horses. Gingivitis, periodontitis, and dental caries can all lead to weakness and decay of teeth that can result in tooth fractures, necessitating exodontia [34].

Although reports of severe, systemic complications following exodontia in horses are uncommon, a few have been reported in the literature in the last several decades and revolve around the diagnosis of post-operative endocarditis [35]. In most reports of equine bacterial endocarditis, no primary source of infection is identified (however, several horses with tricuspid valve lesions had septic jugular vein thrombophlebitis as the primary cause) [35]. Although descriptions of horses with bacterial endocarditis resulting definitively from periodontal or gingival disease have not been reported, it is possible that bacteremia from these conditions could predispose horses to infection. In a 2016 study performed by Kern et al., bacteremia before, during, and after exodontia was described in a group of 20 adult horses. In that study, it was reported that $90 \%$ of the sampled patient population developed a positive blood culture result during at least one time point 
throughout the course of surgery [36]. To the author's knowledge, Kern et al. were the first and only group to publish data on bacteremia in horses, post-exodontia. This finding confirmed the concept that horses also sometimes develop transient bacteremia during tooth extraction, as had been demonstrated in humans and veterinary species $[33,36]$.

Periodontal disease and resulting bacteremia are known to lead to renal, hepatic, or cardiac disease in human and canine species and, therefore, they may also be a risk factor for horses developing these undesirable sequelae [37]. Specifically, SemedoLemsaddek et al. (2016) described a conclusive cause-and-effect relationship between enterococcal infective endocarditis following periodontal disease in dogs [38]. Although endocarditis is a rare complication, effective treatment options are limited and it is often difficult or impossible to treat or resolve it in veterinary species. Since this has been reported rarely in horses, it is logical to use caution in equine patients that have compromised health status when planning elective and necessary equine dental procedures. Examples of equine-specific compromised health status that should be considered in this regard include: pituitary pars intermedia dysfunction (PPID), advanced age, emaciation, severe endoparasitism, protein-energy malnutrition, and immunodeficiency disorders such as common variable immunodeficiency (CVID) [3941].

Equine dental procedures, including invasive extractions, are being employed more commonly in the last decade due to advancements in equipment and increased recognition of the importance of oral health (and its relationship to systemic health). In parallel with increased interest in equine dentistry, the discovery and characterization of a novel syndrome, equine odontoclastic tooth reabsorption and hypercementosis (EOTRH), 
was recently reported [42]. EOTRH is a chronic, painful, degenerative, debilitating disease of the canine and incisor teeth affecting middle-aged to older horses that is currently best treated by the removal of affected teeth, thus maintaining comfort and function [42]. Although not originally recognized as part of the condition, recent work by Sykora et al. demonstrated that there may be an association with oral microbiome changes and EOTRH. Specifically, some novel Treponema and Tannerella spp. were isolated in association with EOTRH-related periodontal disease [34].

Due to the increased extent to which dental extractions are being performed by equine veterinarians in recent years (facilitated by improved equipment, more expertise, and better recognition of disease), it seems likely that more complications will be encountered/reported in horses associated with the post-surgical period following exodontia. Improved follow-up examinations on cases and communications between veterinarians and owners may also lead to an increase in reported cases, as associations and diagnoses are made based on history of recent dental procedure. The complications related to bacteremia seen in dogs (endocarditis, etc.) may become more prevalent in horses due to an increase in the number of dental extraction procedures being attempted and performed.

\section{Using Next Generation Sequencing to Characterize Microbiomes and Uncultivable Bacteria}

Recently, NGS approaches (polymerase chain reaction [PCR] technology) can be used to identify bacteria based on their $16 \mathrm{~S}$ rRNA signature. In order to perform this type of analysis, samples of biological material must be collected and total DNA extracted using a combination of mechanical and enzymatic disruption [43, 44]. Once the DNA has 
been extracted, comparison of genomic regions between collected samples and reference data is undertaken. When choosing genetic markers for phylogenetic analysis, the $16 \mathrm{~S}$ rRNA gene is chosen due to its stability and the fact that it is present in all Bacteria and Archaea [43]. This gene is approximately 1,550 base pairs long and is composed of nine hypervariable regions and several more highly conserved regions. Within the hypervariable regions, sequence differences characterizing specific organisms allow for the taxonomic identification of the bacteria present in a sample. This method, 16S rRNAbased sequencing, is the current gold standard for sequence-based bacterial analysis [43].

Detecting bacteria based on their 16S rRNA signature does not require bacterial culturing and allows for both identification and characterization of bacteria in various samples, such as surface swabs, gastrointestinal contents, and blood [43, 45]. The 16S rRNA signature detection does not distinguish between living bacteria, dead bacteria, and bacterial products or fragments containing RNA [43]. The microbiome present in the bloodstream of the horse has not been well studied in relation to blood culture, but based on human medical literature, it is assumed that a significant amount of the bacteria comprising the microbiome present in horse blood are not detectable with conventional techniques.

Although bacteremia (as characterized with blood culture) [35] has been demonstrated in horses undergoing exodontia, there are currently no reports (to the authors' knowledge) that characterize microbiome analysis of blood samples obtained before and after exodontia [36]. Reliance on blood culturing approaches to identify contaminating bacteremia is an insensitive method because a majority of bacteria are uncultivable and antimicrobial components of blood are inhibitory to those bacterial 
species that are cultivable in vitro [36]. Moreover, the number of bacteria present in the bloodstream following minor dental procedures is very small. For example, only approximately $10 \mathrm{CFU} / \mathrm{mL}$ were reported in the blood for approximately 30 minutes following minor procedures in people [33]. PCR-based NGS approaches have recently been used to analyze the population of bacteria associated with dental caries in horses [46]. The 16S rRNA gene sequence analysis has been used to characterize equine dental caries, as it has been proposed that at least $50 \%$ of oral bacteria are presently regarded as non-cultivable using current standard techniques [46].

\section{Use of Antimicrobials in the Context of Equine Dentistry}

Although bacteremia has been reported in horses during and after exodontia, the use of systemic antimicrobial drugs is inconsistent and there are no current guidelines on when and for how long they should be utilized [36]. In dogs, there has been an association between periodontal disease and histopathological changes in the kidneys, liver, and myocardium [2]. Endocarditis has specifically been associated with periodontal disease in dogs and is proposed to be a potential risk in horses with periodontal disease and exodontia [38]. In the study by Kern et al. it was noted that two horses developed bacteremia (characterized by positive blood culture) after the completion of extraction procedures [36]. Although antimicrobials are not always used in human dentistry, they are sometimes employed under certain circumstances (more invasive procedures or if the patient is immunocompromised). One study demonstrated that dentists were more likely to prescribe prophylactic antibiotics to patients if they are believed to be at risk of developing an infection, including those undergoing invasive oral health procedures such as surgical endodontic therapy or apicectomies, nonsurgical endodontic therapy, and 
extractions, especially surgical extractions and conventional extractions taking more than 5 minutes [47]. It has been observed that patients with a systemic disease, periodontitis, poor oral hygiene, or a smoking habit more commonly received prescriptions for prophylactic antibiotics when undergoing dental surgical procedures [31, 47].

Commonly employed antimicrobials used in equine medicine in this context include amoxicillin, ampicillin, clindamycin, first-generation cephalosporins, and macrolide antibiotics [32, 48]. Bacteremia and right-sided endocarditis have been reported as sequelae of dental extraction in the horse [35]. Serious systemic complications following exodontia have not been commonly reported in the literature so it is logical to question the validity of antimicrobial treatments for exodontia in horses, especially at a time when objective antimicrobial stewardship is so important. Using NGS and identification of bacteria based on their 16S rRNA signature, the population of bacteria present normally in the equine bloodstream and the population of bacteria after exodontia should be further characterized, thus helping guide future antimicrobial use protocols for different types of dental procedures in this species. In the current climate, it is important to remember that, whenever practical "we must develop narrow-spectrum antibiotics, ideally aimed at a single taxon, to avoid the collateral effects of broadspectrum antibiotics on "innocent bystanders" in the microbiota"[49]. 


\section{CHAPTER 2: STUDY}

\section{Hypotheses and Specific Aims}

In this study, we aimed to quantify and identify the bacterial 16S rRNA signature present on the tooth to be extracted and its surrounding gingiva prior to surgery, in the bloodstream prior to surgery, and in the bloodstream one hour after completion of various exodontia procedures undertaken in tranquilized (standing) adult horses. We hypothesized that the bloodstream will contain a significantly higher number and more diverse population of bacteria (as differentiated by $16 \mathrm{~S}$ rRNA signature) one-hour postsurgery, and that identified bacteria will be representative of the population that is detected on the gingiva prior to starting the procedure.

\section{Materials and Methods}

\section{Animals}

The study group consisted of 29 adult horses (with several horses requiring multiple procedures), including 22 geldings and 12 mares. The study population had a mean \pm SD age of $19.4 \pm 5.6$ years (range: 3 to 32 years) and mean \pm SD weight of 479.3 $\pm 107.1 \mathrm{~kg}$ (range: 99.0 to $621.0 \mathrm{~kg}$ ), presented to the University of Missouri Veterinary Health Center for dental examination and dental extraction. There were a variety of breeds, including 7 Thoroughbreds, 7 American Quarter Horses, 3 American Paint Horses, 2 Hanoverians, and one each of the following breeds: Tennessee Walking Horse, Standardbred, Saddlebred, Oldenburg, American Miniature Horse, Haflinger, National Show Horse, Welsh pony, Missouri Fox Trotting Horse, and Arabian. None of the horses had received antimicrobial drugs for at least 1 week prior to presentation. All horses 
received both a physical examination and an oral cavity examination. Oral endoscopic and radiographic examinations were used, if indicated.

\section{Preparation and Medication}

Horses were placed in stocks. The left jugular vein was subjected to aseptic preparation by clipping and scrubbing with $4 \%$ chlorhexidine gluconate that was rinsed using $70 \%$ isopropanol. Immediately following skin disinfection, a blood sample $(20 \mathrm{~mL})$ was collected from the left jugular vein using a vacutainer needle and immediately transferred into two $10 \mathrm{~mL}$ tubes containing ethylenediaminetetraacetic acid (EDTA).

Subsequently, an indwelling intravenous (IV) catheter was placed into the left jugular vein for drug administration and secured with monofilament suture material. For sedation, horses were given a bolus of detomidine hydrochloride ${ }^{\mathrm{a}}$ at $0.01 \mathrm{mg} / \mathrm{kg}$ bodyweight (BWT) IV and butorphanol tartrate ${ }^{\mathrm{b}}$ at $0.01 \mathrm{mg} / \mathrm{kg}$ BWT IV followed by a constant rate infusion (CRI) of detomidine hydrochloride at $0.005 \mathrm{mg} / \mathrm{kg} \mathrm{BWT} /$ hour IV/butorphanol tartrate at $0.005 \mathrm{mg} / \mathrm{kg}$ BWT/hour CRI IV in saline. Prior to administration of local anesthesia, the gingiva adjacent to both the lingual and buccal aspects of extracted teeth was sampled using a sterile cotton swab that was then placed into a semi-solid transport medium. ${ }^{\mathrm{c}}$

Additionally, anesthesia of the mental, infraorbital, mandibular, or maxillary nerves (as appropriate for location of tooth to be extracted) and local infiltration of the gingiva surrounding the diseased tooth were performed using $2 \%$ lidocaine hydrochloride. ${ }^{\mathrm{d}}$ No antimicrobials were given prior to or during extractions. 
The oral extraction of cheek, canine, or incisor teeth was performed in a standardized manner as described elsewhere [50-52]. One hour following delivery of the last tooth and cessation of all surgical manipulations, blood was aseptically drawn from the left jugular catheter. The first $10 \mathrm{~mL}$ of blood were discarded, and the next $20 \mathrm{~mL}$ were collected and transferred into two $10 \mathrm{~mL}$ tubes containing EDTA. All blood samples and gingival swabs collected were immediately frozen until further processing. All dental procedures were performed by the same veterinarian. Horse-owners gave informed consent for their animals' inclusion in this study, which was approved by the institutional Animal Care and Use Committee (MU ACUC\# 9233).

\section{DNA Extraction}

DNA was extracted from $750 \mu \mathrm{L}$ whole blood and dental/gingival swabs using PowerFecal kits ${ }^{\mathrm{e}}$ according to the manufacturer's instructions, with the exception that, rather than performing the initial homogenization of samples using the vortex adapter described in the protocol, samples were homogenized in the provided bead tubes using a TissueLyser $\mathrm{II}^{\mathrm{e}}$ for three minutes at $30 /$ second, before proceeding according to the protocol and eluting with $100 \mu \mathrm{L}$ of elution buffer ${ }^{\mathrm{e}}$. DNA yields were quantified via fluorometry ${ }^{\mathrm{f}}$ using quant-iT BR dsDNA reagent kits. ${ }^{\mathrm{f}}$ As negative and positive controls respectively, blank reagents $(n=10)$ and one mock bacterial community standard ${ }^{\mathrm{g}}$ were $^{-}$ processed alongside experimental samples.

\section{S rRNA Library Preparation and Sequencing}

Extracted blood and gingival swab DNA was processed at the University of Missouri DNA Core Facility. Bacterial 16S rRNA amplicons were constructed via 
amplification of the V4 region of the 16S rRNA gene with universal primers

(U515F/806R) previously developed against the V4 region, flanked by Illumina standard adapter sequences [53, 54]. Oligonucleotide sequences are available at proBase [55]. Dual-indexed forward and reverse primers were used in all reactions. PCR was performed in $50 \mu \mathrm{L}$ reactions containing $100 \mathrm{ng}$ metagenomic DNA, primers $(0.2 \mu \mathrm{M}$ each), dNTPs (200 $\mu \mathrm{M}$ each), and Phusion high-fidelity DNA polymerase (1 U). Amplification parameters were $98^{\circ} \mathrm{C}^{(3 \mathrm{~min})}+\left[98^{\circ} \mathrm{C}^{(15 \mathrm{sec})}+50^{\circ} \mathrm{C}^{(30 \mathrm{sec})}+72^{\circ} \mathrm{C}^{(30 \mathrm{sec})}\right] \times 40$ cycles $+72^{\circ} \mathrm{C}^{(7 \mathrm{~min})}$. Amplicon pools ( $5 \mu \mathrm{L} /$ reaction) were combined, thoroughly mixed, and then purified by addition of Axygen Axyprep MagPCR clean-up beads to an equal volume of $50 \mu \mathrm{L}$ of amplicons and incubated for 15 minutes at room temperature. Products were then washed multiple times with $80 \%$ ethanol, and the dried pellet was resuspended in $32.5 \mu \mathrm{L} \mathrm{EB}$ buffer, incubated for two minutes at room temperature, and then placed on the magnetic stand for five minutes. The final amplicon pool was evaluated using the Advanced Analytical Fragment Analyzer automated electrophoresis system, quantified using quant-iT HS dsDNA reagent kits, and diluted according to Illumina's standard protocol for sequencing on the MiSeq instrument.

\section{Bioinformatics Analysis}

The DNA sequences were assembled and annotated at the MU Informatics Research Core Facility. Primers were designed to match the 5' ends of the forward and reverse reads. Cutadapt (version 2.6; https://github.com/marcelm/cutadapt) was used to remove the primer from the 5 ' end of the forward read [56]. If found, the reverse complement of the primer to the reverse read was then removed from the forward read as were all bases downstream. Thus, a forward read could be trimmed at both ends if the 
insert was shorter than the amplicon length. The same approach was used on the reverse read, but with the primers in the opposite roles. Read pairs were rejected if one read or the other did not match a 5' primer, and an error-rate of 0.1 was allowed. Two passes were made over each read to ensure removal of the second primer. A minimal overlap of three with the 3 ' end of the primer sequence was required for removal.

The Qiime2 [57] DADA2 [58] plugin (version 1.10.0) was used to de-noise, dereplicate, and count ASVs, incorporating the following parameters: 1) forward and reverse reads were truncated to 150 bases, 2) forward and reverse reads with number of expected errors higher than 2.0 were discarded, and 3) chimeras were detected using the "consensus" method and removed. $\mathrm{R}$ version 3.5.1 and Biom version 2.1.7 were used in Qiime2. Taxonomies were assigned to final sequences using the Silva.v132 database [59], using the classify-sklearn procedure.

Hierarchical clustering was performed using an unweighted pair group method with arithmetic mean (UPGMA) approach based on unweighted Jaccard similarities. Similarly, principal coordinate analysis was performed using Jaccard similarities. Clustering approaches were executed using Past3 software [60], downloaded on August 20, 2019. All methods were carried out in accordance with relevant guidelines and regulations.

\section{Statistical analysis}

Univariate data were first tested for normality using the Shapiro-Wilk method. Non-normally distributed data were then tested using a Kruskal-Wallis ANOVA on ranks, followed by post hoc pairwise comparisons using Dunn's method, with 
significance defined by $p<0.05$. Multivariate data were compared using PERMANOVA based on Jaccard similarities, using Past3 software [60].

\section{Results}

Of the 29 horses, most were determined to be healthy $(n=24)$ or affected with paranasal sinusitis $(n=6)$, PPID $(n=3)$, or asthma $(n=1)$. A total of 34 procedures were performed on 29 horses (some horses returned for a second exodontia procedure at least one month after completion of the first). Procedures included those requiring cheek tooth extraction $(n=25)$ and those requiring incisor or canine tooth extraction $(n=9)$.

Justification for exodontia included: apical tooth root abscessation $(n=9)$, slab fracture $(n=9)$, EOTRH syndrome $(n=8)$, infundibular caries $(n=5)$, crown fractures $(n=3)$, and fractured incisive bone $(n=1)$. Retropulsion of teeth was needed for extraction in three cases and standard intra-oral tooth extraction was performed in the remaining 31 cases. Sinus lavage was performed post-procedurally in all cases with comorbid sinusitis. Detailed signalment and procedural information are presented in Table 1.

To qualitatively assess the validity of microbial signatures detected via 16S rRNA sequencing, DNA amplification (quantified by total number of reads for a given sample among a shared sequencing flow cell) was compared between pre- and post-exodontia blood samples, gingival swabs, negative reagent controls, and a commercially available bacterial community standard. As anticipated, the swabs yielded higher sequence numbers than either group of blood samples, and the mock community standard yielded higher coverage, by an order of magnitude, than negative reagent controls and most blood samples (Figure 1). Notably however, five blood samples collected post-exodontia yielded unexpectedly deep coverage, ranging from 187,130 to 669,731 sequences per 
sample. While sequencing coverage is not absolutely quantitative of starting microbial biomass, these results suggested the presence of increased bacterial biomass in a subset of blood samples collected post-exodontia. Moreover, the validity of the remaining blood samples and a few swabs samples that amplified poorly was brought into question.

Recognizing that the differences in sample coverage would likely skew comparisons of bacterial composition, all samples yielding fewer than 1,055 sequences were removed from the following analyses, and the remaining data were rarefied randomly to a uniform read depth of 1,054 reads/sample. The original sequencing coverage of those samples (Figure 2A) is reflective in the hierarchical clustering of samples based on the rarefied dataset, with those same five highly amplified postexodontia blood samples clustering with the gingival swabs, along with two other postexodontia and one pre-exodontia blood samples with lower coverage (Figure 2B). These relationships were also visualized using principal coordinate analysis (PCoA), which demonstrated a similar pattern with the same post-exodontia blood samples clustering close to the gingival swabs (Figure 3). One-way permutational multivariate ANOVA confirmed significant differences between swabs and pre-exodontia blood $(p \leq 0.0001, \mathrm{~F}$ =6.6), swabs and post-exodontia blood $(p \leq 0.0001, \mathrm{~F}=4.7)$, and between pre- and postexodontia blood $(p=0.039, \mathrm{~F}=1.3)$. All three groups were significantly different from negative reagent controls $(p \leq 0.0001 ; \mathrm{F}=2.6,2.6$, and 6.2 for pre- and post-exodontia blood and swabs, respectively). Collectively, we interpreted these results to indicate compositional similarities between the microbial communities present on the gingiva and those detected in post-exodontia blood in a subset of horses, including those whose samples yielded high sequence counts. 
To identify the taxonomies contributing to the differences between swabs and preand post-exodontia blood, data from control samples were removed, and serial ANOVA testing was performed on all detected Amplicon Sequence Variants (ASV). Based on those ASVs returning the 50 lowest $p$ values, hierarchical clustering was repeated and visualized using a heatmap (Figure 4). The same post-exodontia blood samples clustered with the gingival swabs, due to the shared presence of multiple taxa associated with the oral cavity including members of the genera Actinobacillus, Fusobacterium, Leptotrichia, Porphyromonas, Prevotella, Streptococcus, and Veillonella. Notably, these same taxa linking a subset of post-exodontia blood samples to the gingival microbiota represent the dominant taxa in the gingival microbiota (Figure 5). Thus, we interpreted the extremely high coverage selectively observed in a subset of post-exodontia samples, and compositional similarities between those samples and the oral cavity microbiota, as compelling evidence of bacteremia resulting from translocated gingival microbiota in horses undergoing exodontia procedures. 


\section{CHAPTER 3: DISCUSSION}

To the authors' knowledge, there have been no previous characterizations of an equine blood microbiome, either in health or disease. The present study is the first to provide information about the equine blood microbiome in adult horses before and after exodontia. 16S rRNA gene profiling has consistently yielded greater microbial diversity in samples with an anticipated low microbial biomass (such as amniotic fluid and blood) than appreciated based on culture-dependent methods [28]. We adopted an approach that had been successfully employed to improve $16 \mathrm{~S}$ rRNA sequencing in several types of samples, including murine blood [61]. The method entailed increasing the PCR cycle number during library preparation from 25 to 40 cycles and was highly effective in the present study, yielding detection of many ASVs in blood of horses both before and after exodontia. While the requisite reagent controls yielded greater coverage than many of the blood samples, the marked increases observed in a subset of post-exodontia samples, along with the compositional similarity to oral microbiota in those same samples, demonstrate the utility of increased cycle number for similar samples with low microbial biomass.

Post-exodontia bacteremia has been well documented in non-equine species and is associated with various potential health complications [1, 2, 28, 31-33, 36, 48, 62-64]. Results of earlier studies have varied based on the specific surgical treatment undertaken, method used for bacterial identification, immune system responsiveness, and whether antimicrobial drugs were present in sampled blood at the time of collection [33]. In most instances, distant site bacterial infections (such as bacterial endocarditis) were attributed to bacteria originating from the oral microbiome (including Streptococcus mitis and 
Streptococcus oralis in the human medical context) [9]. Although there have been few reports of distant site infections associated with post-exodontia bacterial showering in horses, implicated pathogenic bacteria were also likely derived from oral cavity microbiota $[65,66]$. Results of the present study show that the 16S rRNA signatures of bacteria present at the gingiva in proximity to an extracted (diseased) tooth are similar to those detected in the blood following exodontia. Moreover, the results of the present study indicate that bacteremia by oral commensal bacteria may still be evident one hour following exodontia, in some cases.

Prolonged bacteremia post-exodontia would likely increase the chances of adverse health complications, especially in immunocompromised individuals. If the immune system is ineffective at clearing bacteria from the bloodstream for up to one hour post-operatively from an exodontia procedure, bacteria have greater opportunity to become disseminated throughout the body and potentially colonize and infect a remote location. Since the present study is the first to report bacteremia up to one hour postoperatively, there is a need to examine this phenomenon further to evaluate how long it could potentially last and to investigation the applicability of systemic prophylactic or continued post-operative antimicrobial therapy.

Whereas the human oral microbiome (reportedly the most extensively studied human microflora) has been extensively characterized $[9,11]$, only a few descriptions of the equine oral microbiome have been published [6, 34, 46, 67]. Approximately 500 prevalent bacterial species have been identified in the human oral cavity based on culture-independent molecular methods [9]. Specifically, using culture-independent $16 \mathrm{~S}$ 
rRNA gene clonal analyses, a majority of bacterial species present in the oral cavity are currently deemed uncultivable $[10,11,68]$.

Uncultivable bacteria have been increasingly identified due to the widespread application of $16 \mathrm{~S}$ rRNA gene cloning and sequencing methods to identify microorganisms in natural samples [69]. The advent of such techniques has revealed an extensive and somewhat unsuspected diversity within bacterial phyla thought to be previously well known [69]. It is estimated that less than $2 \%$ of bacteria can be cultured in the laboratory using conventional techniques and in vitro culturing techniques and conditions may not allow growth of all the bacteria in a sample [70]. It is also possible that in vitro conditions do not replicate environmental bacterial cytokine networks that mediate signaling (may be important for coordinating growth) and biofilm formation [70]. As science progresses and more is learned about individual bacterial composition and requirements for growth, some of the uncultivable bacteria today may be readily cultivable in the future using novel techniques and by targeting specific bacterial requirements.

Earlier investigations of the equine oral cavity microbiota using bacteriological culturing methods showed that Gram positive cocci (mainly Streptococci, Micrococci, and starch hydrolyzers) represent prevalent colonizers in healthy horses [46, 71, 72]. Both Gemella spp. and Actinobacillus spp. are also frequently associated with periodontal health in horses $[34,36,46]$. Corynebacterium spp. and Moraxella spp. have also been identified in the oral cavity of healthy horses [46]. In another study, Actinobacillus spp. and an unclassified Pasteurellaceae sp. were the most abundant taxa present in healthy subgingival plaque samples from horses [67]. In that study, Gammaproteobacteria, 
Firmicutes, and Bacteroidetes (with Treponema, Tannerella, and Porphyromonas species detected at low levels) represented the predominant bacterial phyla identified in the healthy equine subgingival microbiome $[6,67]$.

16S rRNA gene sequencing was used to show that periodontitis is associated with disruption of the oral cavity microbiota (dysbiosis) in horses [6]. Whereas bacteria in the healthy oral cavity included Prevotella spp., Veillonella spp., Gemella spp., and Actinobacillus spp., both Tannerella and Treponema genera were significantly increased when periodontitis was identified [6]. 16S rRNA PCR was also used to show that acidogenic and aciduric bacteria, including Streptococcus species, are associated with peripheral caries in horses, as has been reported in other species [43]. Novel red complex bacteria, Treponema and Tannerella species, were also identified through their DNA signatures from the gingiva of EOTRH-affected horses [34]. It was determined that Treponema and/or Tannerella DNA was present in 100\% of horses with periodontitis and only $52.2 \%$ of healthy horses, suggesting that the aforementioned bacterial species have a pathogenic role in the equine mouth [34]. In another study, 18 of 20 horses developed positive blood cultures following exodontia and, in some of those horses, gingival elevation alone resulted in bacteremia [36]. The most commonly identified bacteria on blood culture in that study were Streptococcus spp., Actinomyces spp., Fusobacterium spp., and Prevotella spp.; bacterial genera isolated from swab samples of extracted teeth were similar to those detected in the blood, emphasizing that bacteremia resulted from translocation of oral cavity bacteria [36]. However, it should also be noted that results of bacteriological culturing likely underestimate the extent of bacteremia because most oral cavity bacteria are presently uncultivable [73]. 
Collectively, these studies demonstrate commonalities in oral microbiota composition between diverse species (human, canine, and feline) and that the equine oral microbiome appears to be broadly similar at the taxonomic level of genus and higher [6, 67]. Consistent with previous publications, predominant genera that were identified in the oral cavity of horses in the present study included Actinobacillus, Fusobacterium, Leptotrichia, Porphyromonas, Prevotella, Streptococcus, and Veillonella. Moreover, these same taxa were identified in the five post-exodontia blood samples that yielded unexpectedly deep coverage (prolonged bacterial DNA presence). Four of those horses were also affected with sinusitis, suggesting that post-exodontia bacteremia may be more significant when exodontia is undertaken in horses with comorbid sinusitis. This may be due to local inflammation already being present in the area, allowing for a leakier blood vessel barrier for bacteria to penetrate, or it could possibly be due to the larger number of bacteria present locally at and around the surgical site. Although sinusitis in this study appeared to have some correlation with increased bacterial 16S rRNA signature retrieval from the bloodstream, a larger number of cases would need to be studied to validate this association. Sinusitis is a commonly encountered clinical sign in horses with periapical tooth root infection due to the close association of several of the maxillary cheek teeth with the sinuses $[52,74]$. Specifically, when dealing with secondary sinusitis, the most commonly afflicted teeth include the first molar $(109,209)$, fourth premolar $(108,208)$, and third premolar $(107,207)$ [74].

The use of 16S rRNA gene cloning and sequencing methods has led to the emerging realization that many diverse bacterial phyla that were previously unrecognized or considered unimportant do play a significant role in some diseases [69]. It is becoming 
increasingly evident that commensal bacteria from the oral cavity microbiome are important in the pathogenesis of post-exodontia complications in people following dental surgery $[11,31,62,68,73]$. Although $16 \mathrm{~S}$ rRNA gene cloning and sequencing methods do not differentiate living bacteria from residual bacterial nucleic acid, even residual microbial DNA (in the absence of viable bacterial cells) can serve as an inflammatory signal via innate immune mechanisms including various Toll-like receptors [75]. In light of the fact that a majority of identified bacteria not readily cultivable, it is not possible to conclude which, if any, of the identified bacteria are playing a clinically important role in the pathogenesis of exodontia-associated disease based solely on their respective $16 \mathrm{~S}$ rRNA signatures [73].

It has long been recognized that bacteremia resulting from either dental infection or dental surgery can lead to distant infection (such as bacterial endocarditis), especially in immunocompromised individuals [35, 38, 48, 76-78]. Disruption of the gingival-blood barrier as a result of disease or surgical intervention potentially facilitates translocation of bacteria and bacterial products into the circulation, potentially leading to systemic diseases [21]. Moreover, there is emerging realization that anaerobic commensal bacteria from the oral cavity might, given access to the circulation, play a role in the pathogenesis of a remarkable and diverse inventory of extra-oral diseases. Various (human) diseases that have been attributed to this phenomenon include diabetes mellitus, respiratory disease, cardiovascular disease, and atheroma [22-24]. Of special interest in this regard is Fusobacterium nucleatum, which has been associated with dental disease, various adverse pregnancy outcomes (chorioamnionitis, preterm birth, stillbirth, neonatal sepsis, and preeclampsia), neoplastic and inflammatory gastrointestinal diseases, and various 
other infections in human patients [24]. In 1998, Socransky et al. defined a set of three species showing strong associations with periodontal disease and with each other: Porphyromonas gingivalis, Treponema denticola and Tannerella forsythia which was further verified using PCR technology in 2012 [79, 80]. Although it remains to be seen whether these oral cavity anaerobic commensals might contribute to systemic disease in a hitherto unrecognized manner in horses, the fact that periodontal disease is very common in aging horses and that Fusobacteria were prominently identified in post-exodontia blood in the present study suggests that parallel equine studies should be undertaken [81]. Considering potential bacterial associations between systemic diseases known to be associated with dental disease (i.e. endocarditis, adverse pregnancy outcomes) using NGS may reveal patterns similar to what has been observed in human medicine.

The extent to which post-procedural bacteremia persists has not been extensively reported. In one (human) investigation it was reported that viridans group streptococci were rapidly (within 10 minutes) eliminated from 42 of 46 patients undergoing various oral surgical procedures [33]. In one equine study, two blood samples yielded positive cultures following exodontia (samples obtained 10 minutes after the termination of surgery), providing evidence for short term persistence of bacteremia [36]. Those authors speculated that persistence of bacteremia could have resulted from a greater number of bacteria (quantitative bacterial counts were not performed) or a result of immune function variations between individual horses (two horses in that study were bacteremic prior to the surgical procedure) [36]. Results of earlier work in other species suggest that intravascular bacteria are rapidly cleared from the circulation by the reticuloendothelial system (within 10-20 minutes) [82]. Our results show that significant post-exodontia 
bacteremia is still evident at 60 minutes following conclusion of surgery in some horses. The immune status of the horses in this study was not examined, but future investigations could incorporate an evaluation of the immune system (such as routine hematology and PPID testing) for horses receiving exodontia surgery. Specialized tests of innate immune system function are available to veterinarians at specialized laboratories that involve assessing neutrophil function. Further studies might also evaluate additional time points beyond one hour for evidence of longer-persisting bacteremia.

The use of prophylactic antimicrobials in the perioperative period is restricted to more invasive dental procedures in human dentistry, especially for individuals affected with immunocompromising comorbidities or those with cardiac disease or implants [83, 84]. Antimicrobials are used under the assumption that they do not prevent bacteremia but inhibit bacterial propagation and bacterial adherence to tissues/implants [83, 84]. Specific guidelines for antimicrobial use in horses receiving exodontia have not been published. Results of the present study showing marked post-exodontia bacteremia persisting for at least one hour suggest that antimicrobial use might be important in this setting, especially for immunocompromised horses. In the present study, age and immune status (horses previously diagnosed with PPID) were not identified as positive risk factors for post-operative bacteremia. In future studies, it may be relevant to examine a larger cohort of animals and perform PPID screening tests to look for associations of age and immune status.

Using only a solitary time point for blood sampling post-exodontia (one hour post-operatively) was a limitation of this study and the results imply significant postprocedural bacteremia may persist beyond this timeframe and is deserving of further 
investigation. Although time expended with each exodontia was not measured, it is reasonable to assume that difficult extractions requiring more time could be associated with increased post-procedural bacteremia when compared with more expeditiously concluded procedures. Other limitations include the limited number of cases and the lack of age-matched controls. Blood microbiome results do not necessarily reflect a normal population as all recruited horses were affected with dental disease necessitating exodontia and pre-exodontia blood microbiomes may have been influenced by the presence of dental infection. It should be emphasized that 16S rRNA gene sequencing results are relative, meaning that the actual quantity of bacteria in a given sample is uncertain [85]. It is also possible that each $16 \mathrm{~S}$ rRNA gene may not amplify with equal efficiency during PCR reactions due to differential primer affinity and GC content and taxonomy assignment is conditional upon the completeness of reference databases [85]. Moreover, multiple studies have demonstrated that increased PCR cycle numbers during library preparation are likely to increase the error rate and potentially introduce bias [61, $86,87]$. The use of such methods should therefore be based on the sample type and goals of the study, and results interpreted appropriately.

\section{Conclusions}

The results of this study affirm that bacteremia resulting from translocated oral cavity commensals occurs in horses following dental extraction. Additionally, postexodontia bacteremia is still evident in some individuals for up to one hour, which is much longer than had been previously documented. These results include the first extensive documentation of a blood microbiome based on $16 \mathrm{~S}$ rRNA gene sequencing in adult horses. The extent of post-exodontia bacteremia, especially as pertains to 
uncultivable commensal bacteria and their propensity to contribute to extra-oral disease, is deserving of further investigation in horses. 


\section{ILLUSTRATIONS}

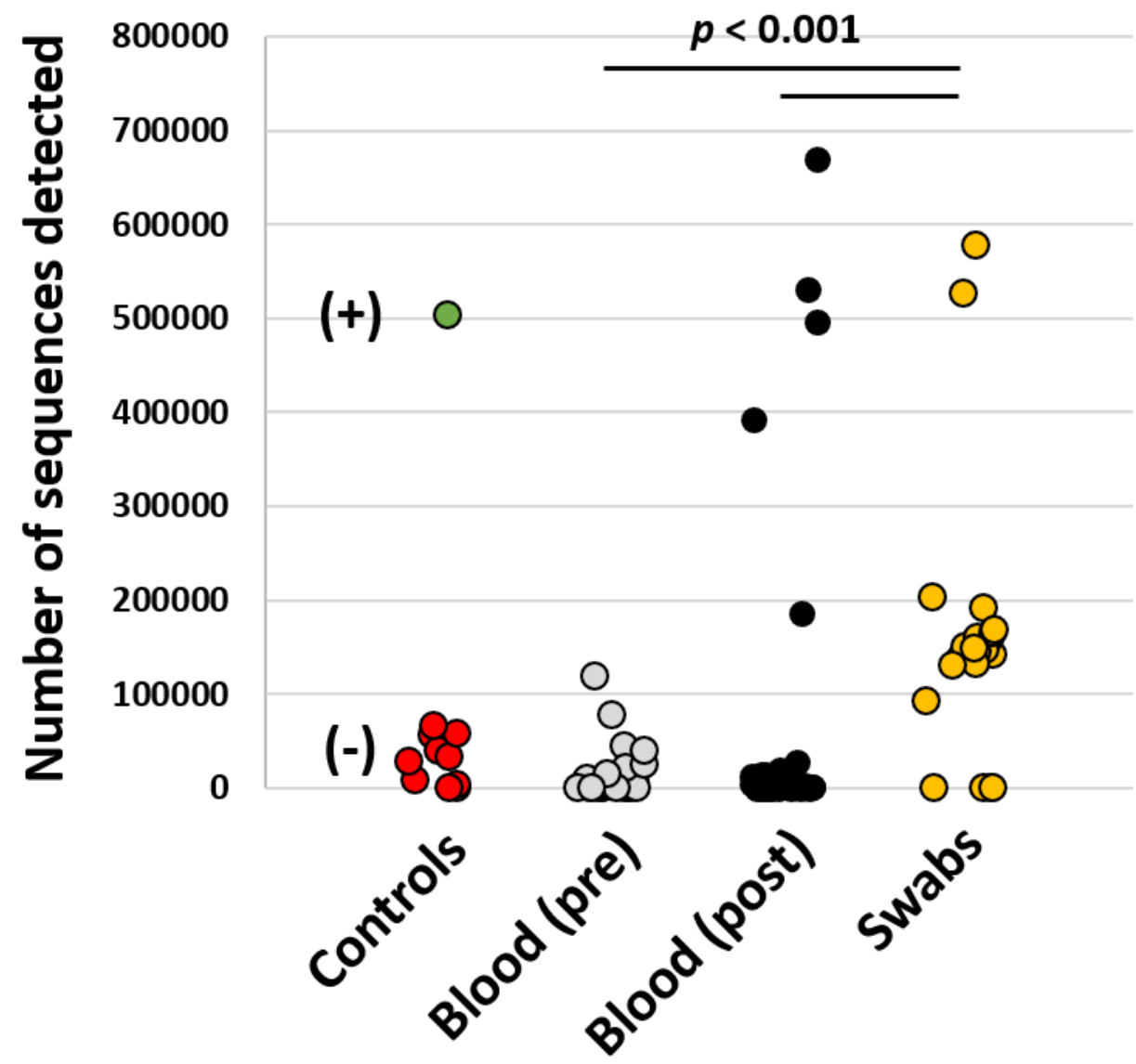

Figure 1: Dot plots showing the total number of 16S rRNA amplicon sequences resulting from amplification and sequencing on a shared flow cell, of negative (-) and positive (+) controls, peripheral blood collected aseptically pre- and post-exodontia procedure, and dental/gingival swabs. 


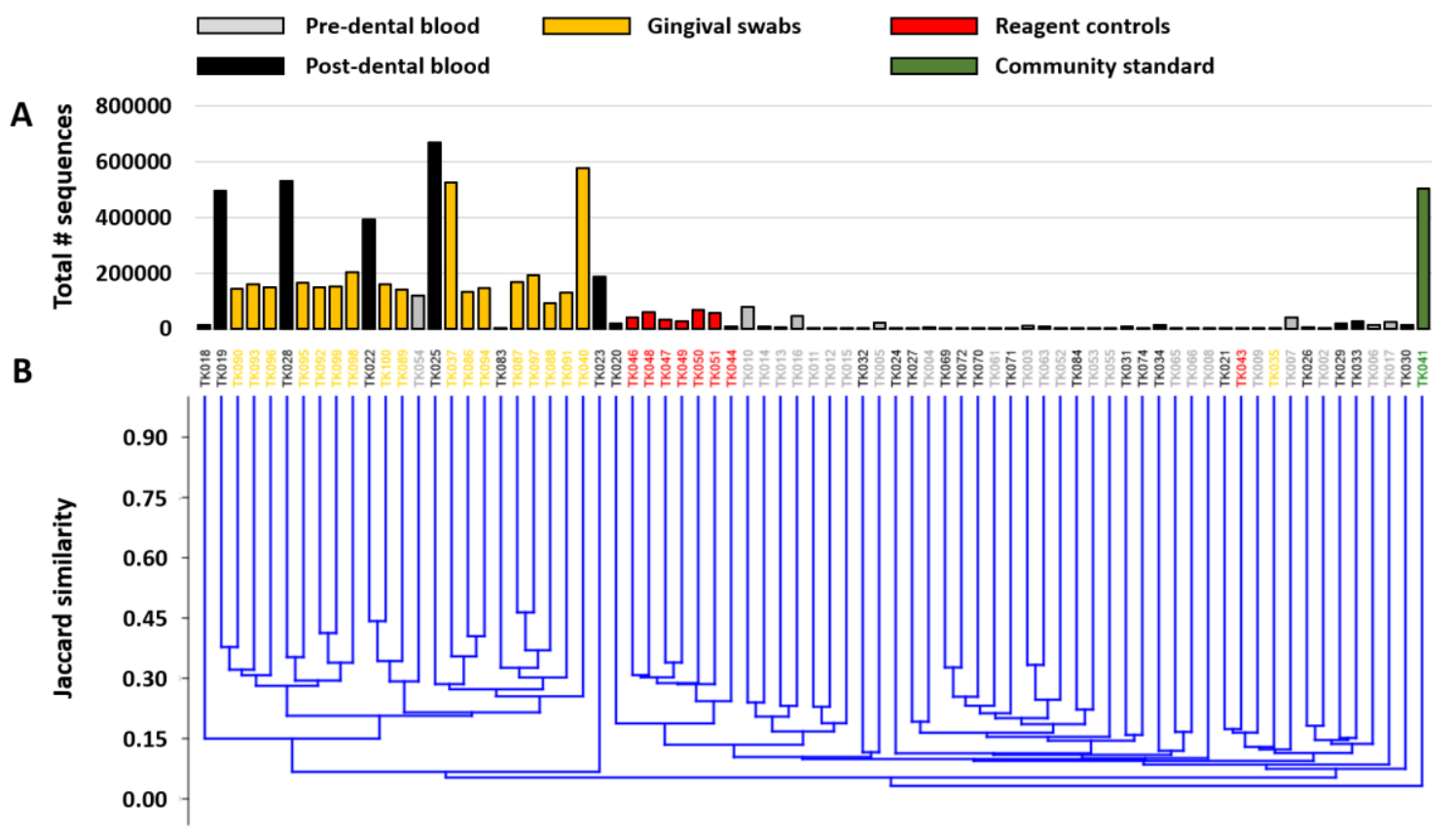

Figure 2: Bar chart showing sample coverage in those samples yielding $>1054$ sequences, legend at top (A), and a dendrogram generated from those data, rarefied to a uniform coverage of 1054 sequences/sample (B). 


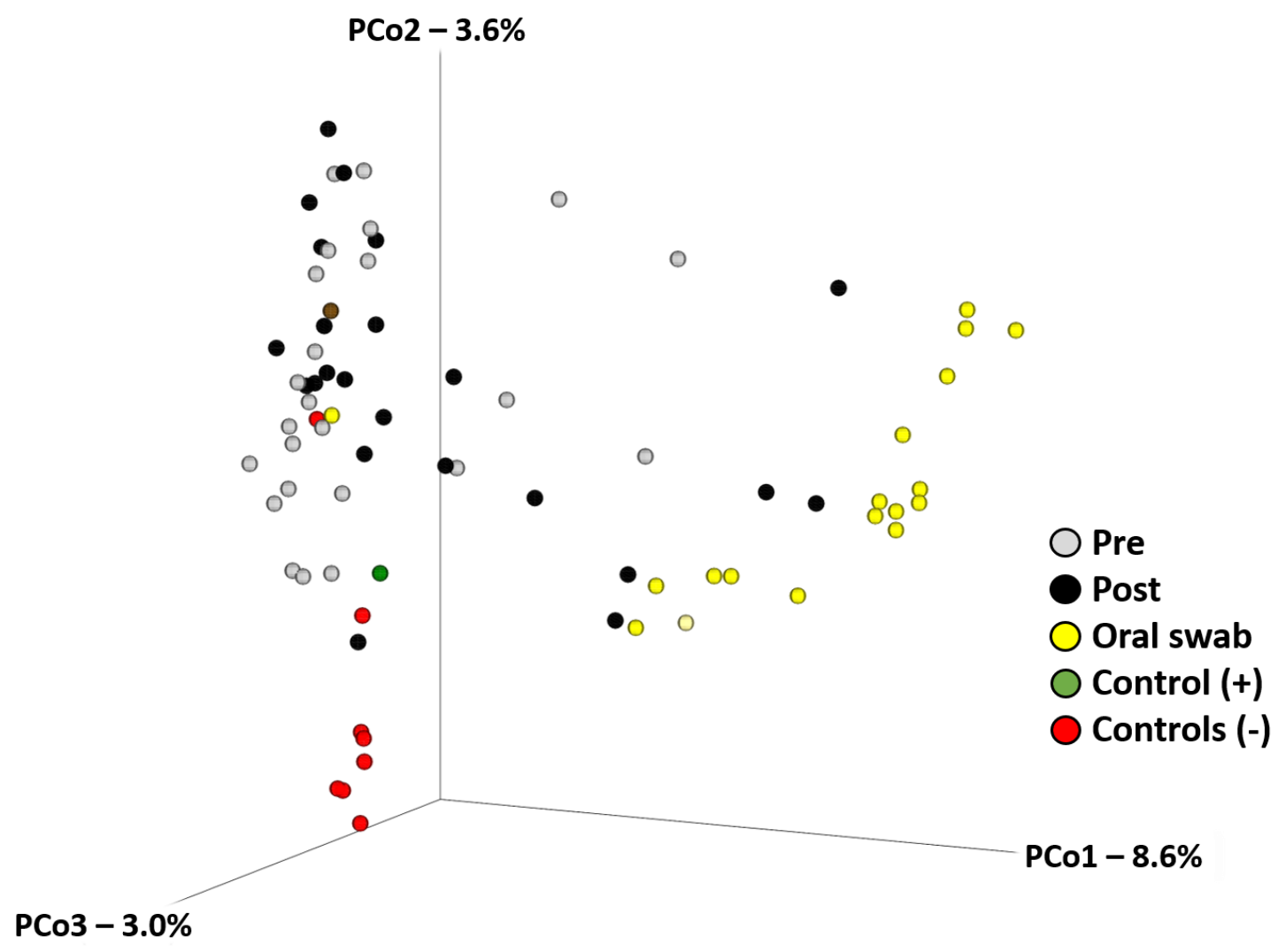

Figure 3: Principal coordinate analysis based on Jaccard similarities and generated using a rarefied dataset (1054 sequences/sample). 


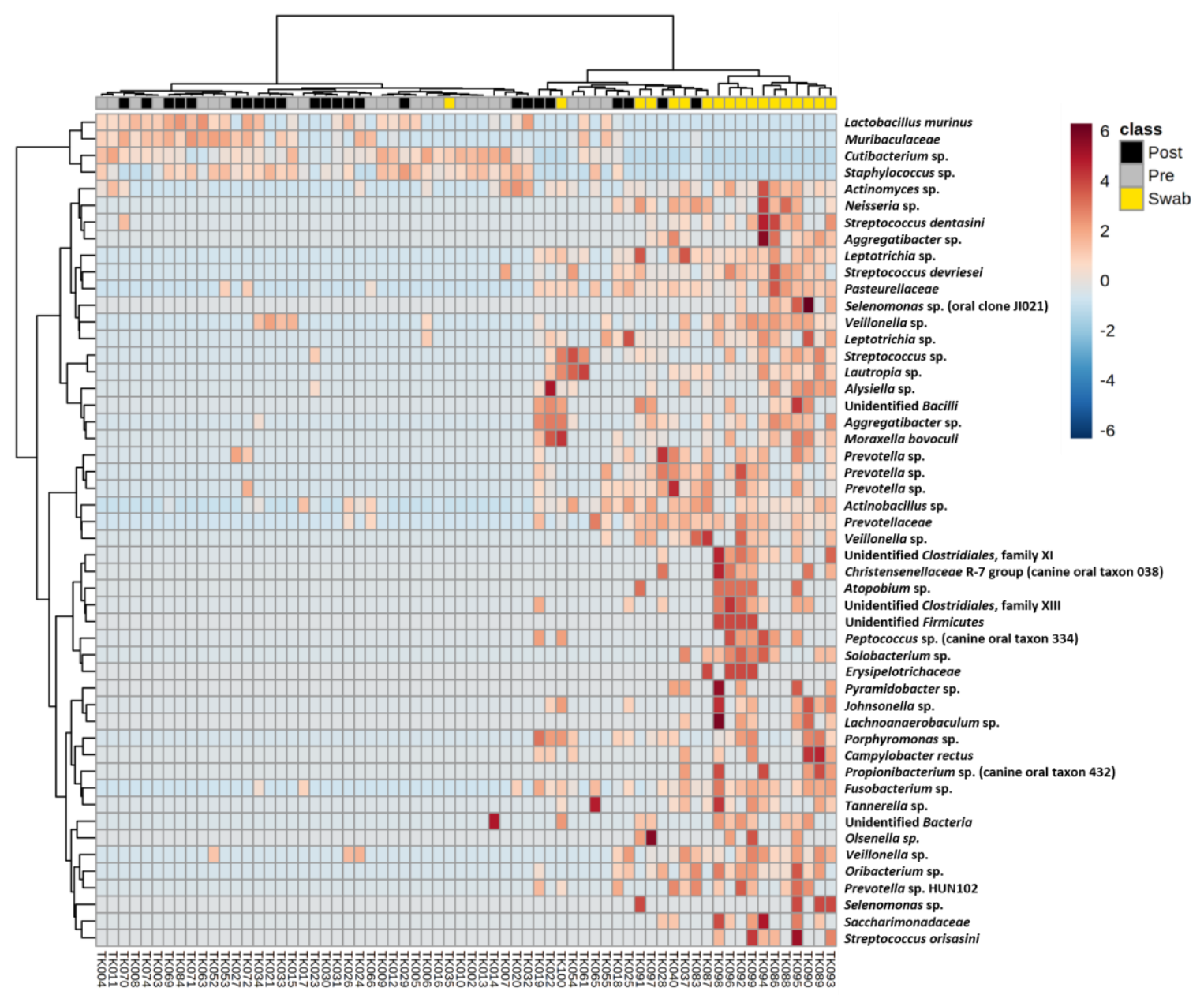

Figure 4: Heatmap generated via hierarchical clustering of samples based on the relative abundance of the 50 ASVs yielding the lowest $p$ values following ANOVA of all ASVs comparing pre- and post- exodontia blood and swabs. 


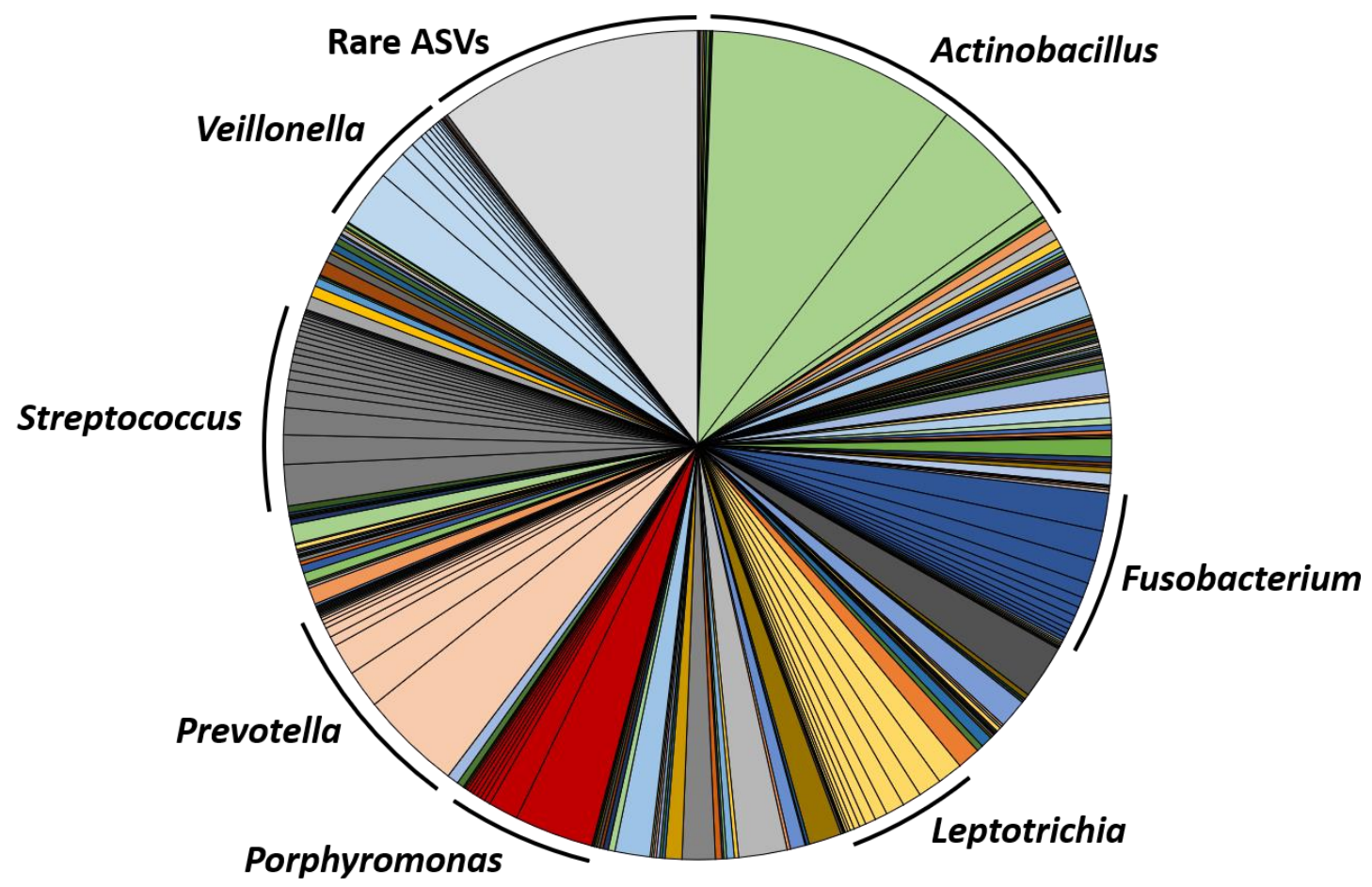

Figure 5. Pie chart showing the mean relative abundance of ASVs detected in the gingival swabs, with dominant genera labeled. The grey portion represents a total of 8544 rare ASVs, comprising roughly $10 \%$ of any given sample. 


\section{TABLES}

\begin{tabular}{|c|c|c|c|c|c|c|c|}
\hline \multirow{2}{*}{$\begin{array}{l}\text { Patient } \\
\text { Horse } 1^{\text {a }} \\
\end{array}$} & \multirow{2}{*}{\begin{tabular}{|l} 
Tooth/Teeth \\
111
\end{tabular}} & \multirow{2}{*}{\begin{tabular}{|l} 
Diagnosis \\
Slab fracture 111 \\
\end{tabular}} & \multirow{2}{*}{$\begin{array}{l}\text { Procedure } \\
\text { Removal of } 111\end{array}$} & \multicolumn{2}{|c|}{ Healthy PPID } & \multicolumn{2}{|c|}{ Asthma Sinusitis } \\
\hline & & & & $\mathrm{x}$ & & & \\
\hline Horse $1^{\mathrm{b}}$ & 110,211 & Recessed and missing parts of crown 110, slab fracture 211 & Removal of 110 and removal of palatal slab of 211 & $\mathrm{x}$ & & & \\
\hline Horse $2^{\mathrm{a}}$ & Mandibular incisors & EOTRH & Removal of mandibular incisors & $\mathrm{x}$ & & & \\
\hline Horse $2^{\mathrm{b}}$ & Maxillary incisors & EOTRH & Removal of maxillary incisors & $\mathrm{x}$ & & & \\
\hline Horse 3 & 109,209 & Nasosinus fistula, sinusitis, apical abscess 109,209 , nasal cyst & Removal of 209,109 , trephination and sinus lavage & & & $\mathrm{x}$ & $\mathrm{x}$ \\
\hline Horse 4 & $202,203,302,303$ & EOTRH & Removal of $202,203,302,303$ & & $\mathrm{x}$ & & \\
\hline Horse 5 & 209 & Slab fracture 209 & Removal of 209 & $\mathrm{x}$ & & & \\
\hline Horse 6 & 209,210 & Fractured 209, sinusitis, apical abscess 210 & Removal of 209,210 , rostral maxillary lavage & & & & $\mathrm{x}$ \\
\hline Horse 7 & 108,109 & Apical abscessation 108, blunted roots 109 & Removal of 108,109 & $\mathrm{x}$ & & & \\
\hline Horse 8 & 208,209 & Apical abscessation, sinusitis & Removal of 208,209 , trephanation and sinus lavage & & & & $\mathrm{x}$ \\
\hline Horse 9 & 204,404 & EOTRH & Removal of 204,404 & & $\mathrm{x}$ & & \\
\hline Horse 10 & Maxillary incisors & EOTRH & Removal of maxillary incisors & $\mathrm{x}$ & & & \\
\hline Horse 11 & 209,210 & Slab fracture 209,210 & Retropulsion of 209,210 and sinus lavage & & & & $\mathrm{x}$ \\
\hline Horse 12 & 108 & Infundibular caries & Removal of 108 & $\mathrm{x}$ & & & \\
\hline Horse 13 & 108 & Sinusitis of the frontal and maxillary sinus (right sided), associated with 108 & Removal of 107,108 caps and sinus lavage & & & & $\mathrm{x}$ \\
\hline Horse 14 & 310,410 & Slab fractures 310,410 & Attempted retropulsion of 410 - failed & $\mathrm{x}$ & & & \\
\hline Horse 15 & 111,211 & Slab fractures 111,211 & Removal of 111 & $\mathrm{x}$ & & & \\
\hline Horse 16 & $107,108,209$ & Apical root abscessation 107, 108, 209 & Removal of $107,108,209$ & $\mathrm{x}$ & & & \\
\hline Horse 17 & $101,102,103$ & Traumatic fracture of incisive bone and tooth roots & Removal of $101,102,103$ & $\mathrm{x}$ & & & \\
\hline Horse 18 & 108 & Crown fracture with periodontal disease & Removal of 108 & $\mathrm{x}$ & & & \\
\hline Horse 19 & 209 & Infundibular caries & Removal of 209 & $\mathrm{x}$ & & & \\
\hline Horse $20^{\mathrm{a}}$ & Maxillary incisors & EOTRH & Removal of maxillary incisors & $\mathrm{x}$ & & & \\
\hline Horse $20^{b}$ & 308,409 & Apical abscessation 308,409 & Removal of 308,409 & & $\mathrm{x}$ & & \\
\hline Horse $21^{\mathrm{a}}$ & Maxillary incisors & EOTRH & Removal of maxillary incisors & $\mathrm{x}$ & & & \\
\hline Horse $21^{b}$ & Mandibular incisors & EOTRH & Removal of mandibular incisors & $\mathrm{x}$ & & & \\
\hline Horse 22 & 209 & Infundibular caries, fracture through pulp horn & Removal of 209 & $\mathrm{x}$ & & & \\
\hline Horse 23 & 110 & Slab fracture, periapical abscess 110 & Retropulsion of 110 and sinus lavage & & & & $\mathrm{x}$ \\
\hline Horse 24 & 109, 209 & Apical absessation, periodontal disease 109,209 & Removal of 109,209 & $\mathrm{x}$ & & & \\
\hline Horse 25 & 208 & Infundibular caries & Removal of 208 & $\mathrm{x}$ & & & \\
\hline Horse 26 & 206,306 & Complicated crown fracture with pulp exposure 206, fractured fragment of 306 & Removal of 206, 306 & $\mathrm{x}$ & & & \\
\hline Horse 27 & 301 & Fractured 301 (suspect traumatic) & Removal of 301 & $\mathrm{x}$ & & & \\
\hline Horse $28^{\mathrm{a}}$ & 109,208 & Infundibular caries 109 , slab fracture 208 & Removal of 109 & $x$ & & & \\
\hline Horse $28^{b}$ & 208 & Slab fracture & Removal of 208 & $\mathrm{x}$ & & & \\
\hline Horse 29 & 309,310 & Apical root abscessation 309,310 & Removal of 309,310 & $\mathrm{x}$ & & & \\
\hline
\end{tabular}

Table 1: Table depicting individual horse information including teeth extracted, diagnosis, surgical procedure, and health designation (healthy, affected with PPID, equine asthma, sinusitis). Teeth were numbered using the Modified Triadan System.

${ }^{\mathrm{a}}$ refers to the first visit, ${ }^{\mathrm{b}}$ refers to second visit 


\section{BIBLIOGRAPHY}

1. Genco, R.J. and M. Sanz, Clinical and public health implications of periodontal and systemic diseases: An overview. Periodontol 2000, 2020. 83(1): p. 7-13.

2. DeBowes, L.J., The effects of dental disease on systemic disease. Vet Clin North Am Small Anim Pract, 1998. 28(5): p. 1057-62.

3. Marshall, M.D., et al., A longitudinal assessment of periodontal disease in 52 Miniature Schnauzers. BMC Vet Res, 2014. 10: p. 166.

4. Stella, J.L., A.E. Bauer, and C.C. Croney, A cross-sectional study to estimate prevalence of periodontal disease in a population of dogs (Canis familiaris) in commercial breeding facilities in Indiana and Illinois. PLoS One, 2018. 13(1): p. e0191395.

5. Vos, T., et al., Global, regional, and national incidence, prevalence, and years lived with disability for 301 acute and chronic diseases and injuries in 188 countries, 1990\&\#x2013;2013: a systematic analysis for the Global Burden of Disease Study 2013. The Lancet, 2015. 386(9995): p. 743-800.

6. Kennedy, R., et al., The microbiome associated with equine periodontitis and oral health. Vet Res, 2016. 47: p. 49.

7. Arweiler, N.B. and L. Netuschil, The Oral Microbiota. Adv Exp Med Biol, 2016. 902: p. 45-60.

8. Yamashita, Y. and T. Takeshita, The oral microbiome and human health. J Oral Sci, 2017. 59(2): p. 201-206.

9. Aas, J.A., et al., Defining the normal bacterial flora of the oral cavity. J Clin Microbiol, 2005. 43(11): p. 5721-32.

10. Dewhirst, F.E., et al., The human oral microbiome. J Bacteriol, 2010. 192(19): p. 5002-17.

11. Chen, T., et al., The Human Oral Microbiome Database: a web accessible resource for investigating oral microbe taxonomic and genomic information. Database (Oxford), 2010. 2010: p. baq013.

12. Kinane, D.F., P.G. Stathopoulou, and P.N. Papapanou, Periodontal diseases. Nat Rev Dis Primers, 2017. 3: p. 17038.

13. Walsh, T., et al., Fluoride toothpastes of different concentrations for preventing dental caries. Cochrane Database Syst Rev, 2019. 3(3): p. Cd007868.

14. Chetrus, V. and I. Ion, Dental Plaque - Classification, Formation, and Identification. International Journal of Medical Dentistry., 2013. 17(2): p. 139143.

15. Kolenbrander, P.E., Oral microbial communities: biofilms, interactions, and genetic systems. Annu Rev Microbiol, 2000. 54: p. 413-37.

16. ten Cate, J.M., Biofilms, a new approach to the microbiology of dental plaque. Odontology, 2006. 94(1): p. 1-9.

17. Marsh, P.D., Are dental diseases examples of ecological catastrophes? Microbiology (Reading), 2003. 149(Pt 2): p. 279-294. 
18. Marsh, P.D. and D.A. Devine, How is the development of dental biofilms influenced by the host? J Clin Periodontol, 2011. 38 Suppl 11: p. 28-35.

19. Humphrey, S.P. and R.T. Williamson, A review of saliva: normal composition, flow, and function. J Prosthet Dent, 2001. 85(2): p. 162-9.

20. Tonetti, M.S., et al., Principles in prevention of periodontal diseases: Consensus report of group 1 of the 11th European Workshop on Periodontology on effective prevention of periodontal and peri-implant diseases. J Clin Periodontol, 2015. 42 Suppl 16: p. S5-11.

21. Gulati, M., et al., Essentials of periodontal medicine in preventive medicine. Int J Prev Med, 2013. 4(9): p. 988-94.

22. Allen-Vercoe, E., J. Strauss, and K. Chadee, Fusobacterium nucleatum: an emerging gut pathogen? Gut Microbes, 2011. 2(5): p. 294-8.

23. Field, C.A., et al., Investigation and quantification of key periodontal pathogens in patients with type 2 diabetes. J Periodontal Res, 2012. 47(4): p. 470-8.

24. Han, Y.W., Fusobacterium nucleatum: a commensal-turned pathogen. Curr Opin Microbiol, 2015. 23: p. 141-7.

25. Pietropaoli, D., et al., Definition of hypertension-associated oral pathogens in NHANES. J Periodontol, 2019. 90(8): p. 866-876.

26. Aagaard, K., et al., A metagenomic approach to characterization of the vaginal microbiome signature in pregnancy. PLoS One, 2012. 7(6): p. e36466.

27. Segata, N., et al., Composition of the adult digestive tract bacterial microbiome based on seven mouth surfaces, tonsils, throat and stool samples. Genome Biol, 2012. 13(6): p. R42.

28. Han, Y.W. and X. Wang, Mobile microbiome: oral bacteria in extra-oral infections and inflammation. J Dent Res, 2013. 92(6): p. 485-91.

29. Conrads, G. and I. About, Pathophysiology of Dental Caries. Monogr Oral Sci, 2018. 27: p. 1-10.

30. Okell, C. and T.S. Elliott, Bacteriaemia and oral sepsis with special reference to aetiology of subacute endocarditis. Lancet, 1935. 226: p. 869-872.

31. Takai, S., et al., Incidence and bacteriology of bacteremia associated with various oral and maxillofacial surgical procedures. Oral Surg Oral Med Oral Pathol Oral Radiol Endod, 2005. 99(3): p. 292-8.

32. Meurman, J.H. and P. Hämäläinen, Oral health and morbidity--implications of oral infections on the elderly. Gerodontology, 2006. 23(1): p. 3-16.

33. Heimdahl, A., et al., Detection and quantitation by lysis-filtration of bacteremia after different oral surgical procedures. J Clin Microbiol, 1990. 28(10): p. 2205-9.

34. Sykora, S., et al., Isolation of Treponema and Tannerella spp. from equine odontoclastic tooth resorption and hypercementosis related periodontal disease. Equine Vet J, 2014. 46(3): p. 358-63.

35. Maxson, A.D. and V.B. Reef, Bacterial endocarditis in horses: ten cases (19841995). Equine Vet J, 1997. 29(5): p. 394-9.

36. Kern, I., et al., Bacteraemia before, during and after tooth extraction in horses in the absence of antimicrobial administration. Equine Vet J, 2017. 49(2): p. 178182. 
37. Pereira Dos Santos, J.D., et al., Relation between periodontal disease and systemic diseases in dogs. Res Vet Sci, 2019. 125: p. 136-140.

38. Semedo-Lemsaddek, T., et al., Enterococcal Infective Endocarditis following Periodontal Disease in Dogs. PLoS One, 2016. 11(1): p. e0146860.

39. McGowan, T.W., G.P. Pinchbeck, and C.M. McGowan, Prevalence, risk factors and clinical signs predictive for equine pituitary pars intermedia dysfunction in aged horses. Equine Vet J, 2013. 45(1): p. 74-9.

40. Ireland, J.L. and C.M. McGowan, Epidemiology of pituitary pars intermedia dysfunction: A systematic literature review of clinical presentation, disease prevalence and risk factors. Vet J, 2018. 235: p. 22-33.

41. Muirhead, T.L., et al., The effect of age on serum antibody titers after rabies and influenza vaccination in healthy horses. J Vet Intern Med, 2008. 22(3): p. 654-61.

42. Staszyk, C., et al., Equine odontoclastic tooth resorption and hypercementosis. Vet J, 2008. 178(3): p. 372-9.

43. Tyler, A.D., M.I. Smith, and M.S. Silverberg, Analyzing the human microbiome: $a$ "how to" guide for physicians. Am J Gastroenterol, 2014. 109(7): p. 983-93.

44. Salonen, A., et al., Comparative analysis of fecal DNA extraction methods with phylogenetic microarray: effective recovery of bacterial and archaeal DNA using mechanical cell lysis. J Microbiol Methods, 2010. 81(2): p. 127-34.

45. Païssé, S., et al., Comprehensive description of blood microbiome from healthy donors assessed by 165 targeted metagenomic sequencing. Transfusion, 2016. 56(5): p. 1138-47.

46. Borkent, D., et al., A microbiome analysis of equine peripheral dental caries using next generation sequencing. Equine Vet J, 2020. 52(1): p. 67-75.

47. Stein, K., et al., The use and misuse of antibiotics in dentistry: A scoping review. J Am Dent Assoc, 2018. 149(10): p. 869-884.e5.

48. Ito, H.O., Infective endocarditis and dental procedures: evidence, pathogenesis, and prevention. J Med Invest, 2006. 53(3-4): p. 189-98.

49. Blaser, M.J., The Past and Future Biology of the Human Microbiome in an Age of Extinctions. Cell, 2018. 172(6): p. 1173-1177.

50. Tremaine, W.H. and J. Schumacher, Exodontia. In Equine Dentistry. Third Edition ed. Exodontia, ed. J. Easly, P. Dixon, and J. Schumacher. 2011, Philadelphia: Saunders Ltd. 424.

51. Tremaine, W.H., Oral extraction of equine cheek teeth. 2004. 16(3): p. 151-158.

52. Caramello, V., et al., Equine cheek tooth extraction: Comparison of outcomes for five extraction methods. Equine Vet J, 2020. 52(2): p. 181-186.

53. Walters, W.A., et al., PrimerProspector: de novo design and taxonomic analysis of barcoded polymerase chain reaction primers. Bioinformatics, 2011. 27(8): p. 1159-61.

54. Caporaso, J.G., et al., Global patterns of 16S rRNA diversity at a depth of millions of sequences per sample. Proc Natl Acad Sci U S A, 2011. 108 Suppl 1: p. 4516-22.

55. Loy, A., et al., probeBase--an online resource for $r R N A$-targeted oligonucleotide probes: new features 2007. Nucleic Acids Res, 2007. 35(Database issue): p. D8004. 
56. Martin, M., Cutadapt removes adapter sequences from high-throughput sequencing reads. 2011, 2011. 17(1): p. 3 \%J EMBnet.journal.

57. Bolyen, E., et al., Reproducible, interactive, scalable and extensible microbiome data science using QIIME 2. Nat Biotechnol, 2019. 37(8): p. 852-857.

58. Callahan, B.J., et al., DADA2: High-resolution sample inference from Illumina amplicon data. Nature Methods, 2016. 13(7): p. 581-583.

59. Quast, C., et al., The SILVA ribosomal RNA gene database project: improved data processing and web-based tools. Nucleic Acids Res, 2013. 41(Database issue): p. D590-6.

60. Hammer, O., D.A.T. Harper, and P.D. Ryan, Past: Paleontological Statistics Software Package for Education and Data Analysis. Palaeontol Electron, 2001. 4( 1): p. 9.

61. Witzke, M.C., et al., Influence of PCR cycle number on $16 S$ rRNA gene amplicon sequencing of low biomass samples. J Microbiol Methods, 2020. 176: p. 106033.

62. Olsen, I., Update on bacteraemia related to dental procedures. Transfus Apher Sci, 2008. 39(2): p. 173-8.

63. Potgieter, M., et al., The dormant blood microbiome in chronic, inflammatory diseases. FEMS Microbiol Rev, 2015. 39(4): p. 567-91.

64. Rawlinson, J.E., et al., Association of periodontal disease with systemic health indices in dogs and the systemic response to treatment of periodontal disease. $J$ Am Vet Med Assoc, 2011. 238(5): p. 601-9.

65. Verdegaal, E.J.M.M., et al., A right-sided bacterial endocarditis of dental origin in a horse. 2006. 18(4): p. 191-195.

66. Bartmann, C., et al., Dentogene Sinusitis durch gramnegative Anaerobier beim Pferd. Tierärztliche Praxis. Ausgabe G, Grosstiere/Nutztiere, 2002. 30: p. 178183.

67. Gao, W., et al., In-depth snapshot of the equine subgingival microbiome. Microb Pathog, 2016. 94: p. 76-89.

68. Curtis, M.A., P.I. Diaz, and T.E. Van Dyke, The role of the microbiota in periodontal disease. Periodontol 2000, 2020. 83(1): p. 14-25.

69. Rappé, M.S. and S.J. Giovannoni, The Uncultured Microbial Majority. 2003. 57(1): p. 369-394.

70. Wade, W., Unculturable bacteria--the uncharacterized organisms that cause oral infections. J R Soc Med, 2002. 95(2): p. 81-3.

71. Baker, G.J., Some aspects of equine dental decay. Equine Vet J, 1974. 6(3): p. 12730.

72. Lundström, T., et al., Equine saliva components during mastication, and in vivo $\mathrm{pH}$ changes in the oral biofilm of sound and carious tooth surfaces after sucrose exposure. Acta Vet Scand, 2020. 62(1): p. 21.

73. Epstein, S.S., The phenomenon of microbial uncultivability. Curr Opin Microbiol, 2013. 16(5): p. 636-42.

74. Freeman, D.E., Sinus disease. Veterinary Clinics of North America: Equine Practice, 2003. 19(1): p. 209-243. 
75. Crump, K.E. and S.E. Sahingur, Microbial Nucleic Acid Sensing in Oral and Systemic Diseases. J Dent Res, 2016. 95(1): p. 17-25.

76. Fülöp, T., et al., The Role of Immunosenescence in the Development of AgeRelated Diseases. Rev Invest Clin, 2016. 68(2): p. 84-91.

77. Glickman, L.T., et al., Evaluation of the risk of endocarditis and other cardiovascular events on the basis of the severity of periodontal disease in dogs. J Am Vet Med Assoc, 2009. 234(4): p. 486-94.

78. Parahitiyawa, N.B., et al., Microbiology of odontogenic bacteremia: beyond endocarditis. Clin Microbiol Rev, 2009. 22(1): p. 46-64, Table of Contents.

79. Socransky, S.S., et al., Microbial complexes in subgingival plaque. J Clin Periodontol, 1998. 25(2): p. 134-44.

80. Griffen, A.L., et al., Distinct and complex bacterial profiles in human periodontitis and health revealed by 165 pyrosequencing. Isme j, 2012. 6(6): p. 1176-85.

81. Nuttall, H.E. and P.J. Ravenhill, Prevalence and analysis of equine periodontal disease, diastemata and peripheral caries in a first-opinion horse population in the UK. Vet J, 2019. 246: p. 98-102.

82. Silver, J.G., L. Martin, and B.C. McBride, Recovery and clearance rates of oral microorganisms following experimental bacteraemias in dogs. Arch Oral Biol, 1975. 20(10): p. 675-9.

83. Wilson, W., et al., Prevention of infective endocarditis: guidelines from the American Heart Association: a guideline from the American Heart Association Rheumatic Fever, Endocarditis and Kawasaki Disease Committee, Council on Cardiovascular Disease in the Young, and the Council on Clinical Cardiology, Council on Cardiovascular Surgery and Anesthesia, and the Quality of Care and Outcomes Research Interdisciplinary Working Group. J Am Dent Assoc, 2008. 139 Suppl: p. 3s-24s.

84. Kuong, E.E., et al., Antibiotic prophylaxis after total joint replacements. Hong Kong Med J, 2009. 15(6): p. 458-62.

85. Jo, J.H., E.A. Kennedy, and H.H. Kong, Research Techniques Made Simple: Bacterial 16S Ribosomal RNA Gene Sequencing in Cutaneous Research. J Invest Dermatol, 2016. 136(3): p. e23-e27.

86. Wu, J.Y., et al., Effects of polymerase, template dilution and cycle number on PCR based 16 S rRNA diversity analysis using the deep sequencing method. BMC Microbiol, 2010. 10: p. 255.

87. Sze, M.A. and P.D. Schloss, The Impact of DNA Polymerase and Number of Rounds of Amplification in PCR on 16S rRNA Gene Sequence Data. 2019. 4(3): p. e00163-19.

\footnotetext{
${ }^{a}$ Dormosedan ${ }^{2}$, Orion Pharma Orion Corporation, Espoo, Finland

${ }^{\mathrm{b}}$ Torbugesic $®$, Zoetis Manufacturing and Research, Spain, S.L., Girona, Spain

${ }^{\mathrm{c}}$ Remel, Lenexa, KS, USA
} 
${ }^{\mathrm{d}}$ Hospira, Inc., Lake Forest, IL, USA

${ }^{\mathrm{e}}$ Qiagen, Venlo, Netherlands

${ }^{\mathrm{f}}$ Qubit 2.0, Invitrogen, Carlsbad, CA, USA

g \#D6300, ZymoBIOMICS, Irvine, CA, USA 